\title{
Establishment, validation and evaluation of predictive model for early relapse after R0 resection in hepatocellular carcinoma patients with microvascular invasion
}

\author{
Kai Zhang ${ }^{\dagger}$, Changcheng Tao ${ }^{\dagger}$, Tana Siqin, Jianxiong $\mathrm{Wu}^{*}$ and Weiqi Rong ${ }^{*}$
}

\begin{abstract}
Backgrounds: This is the first study to build and evaluate a predictive model for early relapse after R0 resection in hepatocellular carcinoma (HCC) patients with microvascular invasion (MVI).

Methods: The consecutive HCC patients with MVI who underwent hepatectomy in Cancer Hospital of Chinese Academy of Medical Science from Jan 2014 to June 2019 were retrospectively enrolled and randomly allocated into a derivation $(N=286)$ and validation cohort $(N=120)$ in a ratio of 7:3. Cox regression and Logistic regression analyses were performed and a predictive model for postoperative early-relapse were developed.

Results: A total of $406 \mathrm{HCC}$ patients with MVI were included in our work. Preoperative blood alpha-fetoprotein (AFP) level, hepatitis B e antigen ( $\mathrm{HBeAg}$ ) status, MVI classification, largest tumor diameter, the status of serosal invasion, number of tumors, and the status of satellite nodules were incorporated to construct a model. The concordance index (C-index) was 0.737 and 0.736 in the derivation and validation cohort, respectively. The calibration curves showed a good agreement between actual observation and nomogram prediction. The C-index of the nomogram was obviously higher than those of the two traditional HCC staging systems.

Conclusion: We have developed and validated a prediction model for postoperative early-relapse in HCC patient with MVI after RO resection.
\end{abstract}

Keywords: Hepatocellular carcinoma, Microvascular invasion, Nomogram, Early-relapse, R0 resection

\section{Introduction}

Hepatocellular carcinoma (HCC) is the sixth and fourth most common malignant tumor worldwide and in China, respectively. It is also the fourth and third most common cause of cancer-related death worldwide and in China, respectively [1]. The risk factors for primary HCC

\footnotetext{
*Correspondence: dr_wujx@163.com; dr_rongweiqi@163.com ${ }^{\dagger}$ Kai Zhang and Changcheng Tao contributed equally to this study Department of Hepatobiliary Surgery, National Cancer Center/National Clinical Research Center for Cancer/Cancer Hospital, Chinese Academy of Medical Sciences (CAMS) and Peking Union Medical College (PUMC), No. 17 Panjiayuannanli, Chaoyang District, Beijing 100021, China
}

tumorigenesis include hepatitis B virus (HBV) infection, hepatitis $\mathrm{C}$ virus (HCV) infection, alcoholism, and nonalcoholic fatty liver disease cirrhosis [2]. In China, HCC is mainly caused by HBV infection, which also impacts on the prognosis of $\mathrm{HCC}$ patients. It has been documented that hepatitis $\mathrm{B}$ e antigen ( $\mathrm{HBeAg}$ ) positivity is an independent factor for poor prognosis of patients with HBVrelated HCC [3]. The use of appropriate management strategies for HBV infection could slow down the development of liver cirrhosis and decrease the risk of the postoperative recurrence of HBV-related HCC [4].

c) The Author(s) 2021. This article is licensed under a Creative Commons Attribution 4.0 International License, which permits use, sharing, adaptation, distribution and reproduction in any medium or format, as long as you give appropriate credit to the original author(s) and the source, provide a link to the Creative Commons licence, and indicate if changes were made. The images or other third party material in this article are included in the article's Creative Commons licence, unless indicated otherwise in a credit line to the material. If material is not included in the article's Creative Commons licence and your intended use is not permitted by statutory regulation or exceeds the permitted use, you will need to obtain permission directly from the copyright holder. To view a copy of this licence, visit http://creativeco mmons.org/licenses/by/4.0/. The Creative Commons Public Domain Dedication waiver (http://creativecommons.org/publicdomain/ zero/1.0/) applies to the data made available in this article, unless otherwise stated in a credit line to the data. 
It is well known that liver transplantation, radiotherapy, ablation, and hepatectomy are the main local treatment strategies for primary HCC patients. Liver transplantation has not been extensively used for the treatment of liver cancer due to the limited donor pool and high cost. With the advancements in radiotherapy technologies, HCC patients achieve a good disease control after stereotactic body radiation therapy (SBRT) treatment [5]. However, the radiotherapy is only recommended for patients with unresectable $\mathrm{HCC}$ who are ineligible for liver transplantation $[6,7]$. The treatment efficacy of ablation therapy is commonly comparable with that of surgical resection in HCC patients [8]. However, some studies have revealed that the relapse-free survival (RFS) and overall survival (OS) in patients with a HCC diameter $<2 \mathrm{~cm}$ after receiving ablation therapy are shorter than those receiving surgical resection [9]. In addition, HCC patients with MVI who receive ablation therapy exhibits a higher early-recurrence rate than those receive surgical resection [10]. Therefore, surgical resection is still the main treatment strategy for radical intent in HCC patients.

Although surgery is currently the main local treatment strategy for HCC, its long-term efficacy is unsatisfactory. As reported by a literature, 2-year relapse rate reaches $54 \%$ in patients receiving surgery [11]. Dozens of studies show that predictive models for relapse in HCC patients after the radical surgery have been constructed to guide postoperative adjuvant treatment decision [1214]. Microvascular invasion (MVI) is an independent risk factor for postoperative relapse in all reported predictive models. As reported by previous studies, the incidence rate of MVI confirmed through postoperative pathological examination is $11-60 \%$ [15] and it is $39 \%$ in our hospital [16]. According to the Standard for Diagnosis and Treatment of Primary Liver Cancer [17], MVI is a mass of cancer cells in vascular cavity with adhesion to endothelial cell observed under a microscope, which mainly occurs in portal vein. It is graded according to the count of cancer cells and the distance of MVI to tumor. The emergence of MVI impacts on the OS, and it is also a risk factor for postoperative early-relapse in HCC patients [18]. The prognosis of HCC patients with postoperative early-relapse is worse than that of patients with laterelapse [19]. Therefore, it is an unmet need to identify HCC patients who are more prone to experience postoperative early-relapse. As previously mentioned, predictive models for postoperative early-relapse in HCC patients have been established, however, a predictive model for HCC patients with MVI who are more prone to experience early-relapse has not yet been reported.

In our study, a predictive model for early-relapse in HCC patients with MVI after receiving R0 resection confirmed by postoperative pathological examination was constructed and its diagnostic performance were validated in a validation cohort.

\section{Methods \\ Patient selection}

The consecutive patients who underwent hepatectomy for HCC in Cancer Hospital of Chinese Academy of Medical Science from Jan 2014 to June 2019 were enrolled. The patients were classified into M1 and M2 subgroups on the basis of MVI status according to the Guideline for Standardized Pathological Diagnosis of Primary Liver Cancer [20]. MX defined as the emergence of MVI with unknown MVI classification. HCC patients with MVI were selected for subsequent analyses as following criteria. Inclusion criteria: (1) HCC with MVI confirmed by the postoperative pathological examination; (2) R0 resection confirmed by the postoperative pathological examination; (3) Child-Pugh A liver function prior to surgery; (4) No serious dysfunction of heart, lung or kidney that impacts on prognosis; (5) Eastern Cooperative Oncology Group (ECOG) performance status of 0-1. Exclusion criteria: (1) Death within one month following surgery; (2) No relapse and a follow-up of $<12$ months; (3) Unknown status of relapse; (4) With lymph node metastasis confirmed by postoperative pathological examination. Flow chart for patient screening was shown in Additional file 1: Fig. S1.

RFS was defined as the time from the end of surgery to the relapse/metastasis or the death of any cause. According to the previous studies $[11,21]$ and the rules of clinical follow-up, the patients with a relapse or death within 12 months after surgery were allocated into the earlyrelapse group and those with a relapse or death over more than 12 months postoperatively were allocated into the late-relapse group [19]. The patients without a relapse or death until the follow-up endpoint was also allocated into the late-relapse group.

\section{Statistical analysis}

All enrolled patients were randomly allocated into a derivation cohort (286 patients) and validation cohort in a ratio of 7:3 (120 patients). Variables independently associated with postoperative early-relapse were determined to construct a predictive model in the derivation cohort as following. First step, all clinicopathological variables collected in the present work were included in the univariate Cox model to identify risk factors significantly associated with RFS. Second step, variables identified in the first step were included in the multivariate Cox model to screen independent risk factors associated with RFS. Third step, variables identified in the second step were included in the multivariate Logistic model to determine 
the independent risk factors associated with postoperative early-relapse. Nomogram and web calculator were subsequently performed to established a predictive model for postoperative early-relapse in the derivation cohort.

Model discrimination measured by concordance index (C-index), model calibration measured by calibration plots and clinical practicability of the predictive model measured by decision curve analyses (DCA) were used to evaluate the predictive performance of the nomogram between patients with and without early-relapse in the derivation and validation cohorts. The predictive performance of the constructed model was compared with that of the $8^{\text {th }}$ edition American Joint Committee on Cancer (AJCC) staging system and Barcelona Clinic Liver Cancer (BCLC) staging system in discrimination, calibration, and clinical usefulness.

The optimal cutoff value of the nomogram was determined by maximizing the Youden index based on receiver operating characteristic (ROC) curves. The optimal cutoff value of AJCC and BCLC staging system was also determined based on ROC curves. Sensitivity, specificity, and accuracy of the predictive model, AJCC and BCLC staging system were calculated in the derivation and validation cohorts, respectively.

Categorical data were analyzed using Chi-square test or Fisher exact test. The continuous variables in a normal distribution were analyzed using $t$-test or variance analysis. The variables in a non-normal distribution were analyzed using rank sum test. A P-value less than 0.05 was considered to be statistically significant. Unless otherwise stipulated, test power $(\alpha)$ for multivariate analysis was set as 0.05. EmpowerStats (http://www.empowersta ts.com, X\&Y Solutions Inc., Boston. MA) and R software (Version 3.6.2) were used for statistical analysis and chart plots. All independent variables were screened by collinearity analysis with variance inflation factor (VIF) $\leq 5$.

\section{Results \\ Baseline characteristics of patients in the derivation and validation cohort}

A total of $1320 \mathrm{HCC}$ patients were retrospectively recruited in our work, including 482 patients with MVI (36.5\%). According to the inclusion and exclusion criteria, a total of $406 \mathrm{HCC}$ patients with MVI were selected for subsequent analyses, including 160 patients (39.4\%) having a postoperative early-relapse, 67 patients (16.5\%) having a postoperative late-relapse and 179 patients (44.1\%) without a relapse until the end of follow-up period. One hundred and sixty (39.4\%) and 246 patients (60.6\%) were with early-relapse and non-early-relapse, respectively.
There were 286 patients and 120 patients were randomly allocated into the derivation and validation cohort in a ratio of 7:3, respectively, by using $R$ language caret package with a seed of 2,020,090,873. The majority of clinical characteristics were not statistically different between the derivation and validation cohort, as shown in Table 1. The derivation cohort showed marginally higher proportion of patients with albumin-bilirubin (ALBI) score $\leq-2.60$ compared with the validation cohort ( $\mathrm{P}=0.084$, Table 1$)$. Preoperative albumin (ALB) level was marginally higher in the derivation cohort than that in the validation cohort $(\mathrm{P}=0.077$, Table 1$)$. Preoperative alanine aminotransferase (ALT) level was significantly higher in the derivation cohort than that in the validation cohort $(\mathrm{P}=0.021$, Table 1$)$.

\section{Determination of risk factors associated with postoperative early-relapse}

Next, the univariate and multivariate Cox regression analyses were performed to determine the independent risk factors associated with RFS in the derivation cohort. Total of 6 independent risk factors associated with RFS were identified, including ALBI score, preoperative $\mathrm{HBeAg}$ status, MVI classification, largest tumor diameter, number of tumors and the status of serosal invasion (Table 2). Preoperative blood AFP level and the status of satellite nodule were marginally statistically associated with RFS. As previously reported, blood AFP level is significantly associated with prognosis and early-relapse, and the multinodular tumor commonly develops from MVI in HCC patients [20], therefore, these two variables were further incorporated into the subsequent analysis. Next, the abovementioned 8 variables were included in the multivariable Logistic regression analysis to determine the risk factors associated with postoperative early-relapse. Total of 5 independent risk factors were identified, including preoperative blood AFP level, preoperative HBeAg status, MVI classification, largest tumor diameter and the status of serosal invasion (Table 2). Number of tumors and the status of satellite nodules were marginally statistically associated with early-relapse (Table 2). The previous studies have revealed that both number of tumors and the status of satellite nodules are independent risk factors for postoperative relapse [18, 22], therefore, these two variables were included for the subsequent analysis to establish a predictive model.

\section{Establishment of predictive model for postoperative early-relapse and evaluation of its discriminability and calibration}

Next, the abovementioned 7 risk factors associated with postoperative early-relapse were included to construct the predictive model by using a binary logistic 
Table 1 The clinical characteristics of patients in the derivation and validation cohort

\begin{tabular}{|c|c|c|c|}
\hline Clinical characteristics & Derivation cohort & Validation cohort & P-value \\
\hline Number of patients & 286 & 120 & \\
\hline Gender & & & 0.242 \\
\hline Male & $237(82.87 \%)$ & $105(87.50 \%)$ & \\
\hline Female & 49 (17.13\%) & 15 (12.50\%) & \\
\hline Age (years) & & & 0.458 \\
\hline$\leq 60$ & $199(69.58 \%)$ & $79(65.83 \%)$ & \\
\hline$>60$ & 87 (30.42\%) & $41(34.17 \%)$ & \\
\hline Hypertension & & & 0.233 \\
\hline With & $74(25.87 \%)$ & $38(31.67 \%)$ & \\
\hline Without & $212(74.13 \%)$ & $82(68.33 \%)$ & \\
\hline Diabetes & & & 0.885 \\
\hline With & $46(16.08 \%)$ & $20(16.67 \%)$ & \\
\hline Without & $240(83.92 \%)$ & $100(83.33 \%)$ & \\
\hline Coronary heart disease & & & 0.803 \\
\hline With & $11(3.85 \%)$ & $4(3.33 \%)$ & \\
\hline Without & $275(96.15 \%)$ & 116 (96.67\%) & \\
\hline Smoking history & & & 0.393 \\
\hline With & $125(43.71 \%)$ & $58(48.33 \%)$ & \\
\hline Without & $161(56.29 \%)$ & $62(51.67 \%)$ & \\
\hline Drinking history & & & 0.187 \\
\hline With & $88(30.77 \%)$ & $45(37.50 \%)$ & \\
\hline Without & $198(69.23 \%)$ & $75(62.50 \%)$ & \\
\hline Portal hypertension & & & 0.325 \\
\hline With & $65(22.73 \%)$ & $22(18.33 \%)$ & \\
\hline Without & $221(77.27 \%)$ & $98(81.67 \%)$ & \\
\hline ALBI core & & & 0.084 \\
\hline$\leq-2.60$ & $256(89.51 \%)$ & $100(83.33 \%)$ & \\
\hline$>-2.60$ to $\leq-1.39$ & $30(10.49 \%)$ & $20(16.67 \%)$ & \\
\hline Preoperative ALT level (U/L) & $30.0(13.0-241.0)$ & $26.50(13.00-337.0)$ & 0.021 \\
\hline Preoperative AST level (U/L) & $30.0(13.0-241.0)$ & $26.50(13.00-337.0)$ & 0.104 \\
\hline Preoperative ALB level (g/L) & $44.02 \pm 4.19$ & $43.20 \pm 4.41$ & 0.077 \\
\hline Preoperative serum creatinine level (mg/dl) & $73.35 \pm 14.29$ & $76.21 \pm 13.28$ & 0.061 \\
\hline Preoperative blood glucose level (mmol/L) & $5.62 \pm 1.72$ & $5.50 \pm 1.37$ & 0.489 \\
\hline Preoperative PT (second) & $11.92 \pm 0.95$ & $11.97 \pm 1.07$ & 0.688 \\
\hline Preoperative status of $\mathrm{HBsAg}$ & & & 0.165 \\
\hline Positive & $232(81.12 \%)$ & $90(75.00 \%)$ & \\
\hline Negative & $54(18.88 \%)$ & $30(25.00 \%)$ & \\
\hline Preoperative status of $\mathrm{HBeAg}$ & & & 0.240 \\
\hline Positive & $65(22.73 \%)$ & $21(17.50 \%)$ & \\
\hline Negative & $221(77.27 \%)$ & 99 (82.50\%) & \\
\hline HCV-Ab & & & 0.669 \\
\hline Positive & $20(6.99 \%)$ & $7(5.83 \%)$ & \\
\hline Negative & $266(93.01 \%)$ & $113(94.17 \%)$ & \\
\hline Preoperative LnAFP level & $4.62 \pm 2.95$ & $4.62 \pm 2.95$ & 0.932 \\
\hline Maximum diameter of primary tumor $(\mathrm{cm})$ & & & 0.836 \\
\hline$\leq 5$ & $170(59.44 \%)$ & 70 (58.33\%) & \\
\hline$>5$ & $116(40.56 \%)$ & $50(41.67 \%)$ & \\
\hline Adjacent to large blood vessels & & & 0.770 \\
\hline With & $90(31.47 \%)$ & $36(30.00 \%)$ & \\
\hline
\end{tabular}


Table 1 (continued)

\begin{tabular}{|c|c|c|c|}
\hline Clinical characteristics & Derivation cohort & Validation cohort & P-value \\
\hline Without & $196(68.53 \%)$ & $84(70.00 \%)$ & \\
\hline Adjacent to the diaphragm & & & 0.078 \\
\hline With & $24(8.39 \%)$ & $17(14.17 \%)$ & \\
\hline Without & $262(91.61 \%)$ & $103(85.83 \%)$ & \\
\hline MVI classification & & & 0.263 \\
\hline M1 & $132(46.15 \%)$ & $58(48.33 \%)$ & \\
\hline M2 & $66(23.08 \%)$ & $34(28.33 \%)$ & \\
\hline$M X$ & $88(30.77 \%)$ & $28(23.33 \%)$ & \\
\hline Number of tumors & & & 0.326 \\
\hline 1 & $250(87.41 \%)$ & 109 (90.83\%) & \\
\hline$\geq 2$ & $36(12.59 \%)$ & $11(9.17 \%)$ & \\
\hline Satellite nodule & & & 0.749 \\
\hline With & $49(17.13 \%)$ & 19 (15.83\%) & \\
\hline Without & $237(82.87 \%)$ & $101(84.17 \%)$ & \\
\hline Serosal invasion & & & 0.657 \\
\hline With & $172(60.14 \%)$ & 75 (62.50\%) & \\
\hline Without & $114(39.86 \%)$ & $45(37.50 \%)$ & \\
\hline Preoperative/intraoperative ablation & & & 1.000 \\
\hline With & $7(2.45 \%)$ & $2(1.67 \%)$ & \\
\hline Without & $279(97.55 \%)$ & $118(98.33 \%)$ & \\
\hline Preoperative radiotherapy & & & 0.727 \\
\hline With & $6(2.10 \%)$ & $3(2.50 \%)$ & \\
\hline Without & $280(97.90 \%)$ & $117(97.50 \%)$ & \\
\hline Preoperative interventional therapy & & & 0.924 \\
\hline With & $16(5.59 \%)$ & $7(5.83 \%)$ & \\
\hline Without & $270(94.41 \%)$ & $113(94.17 \%)$ & \\
\hline Postoperative radiotherapy & & & 0.642 \\
\hline With & $47(16.43 \%)$ & $22(18.33 \%)$ & \\
\hline Without & $239(83.57 \%)$ & $98(81.67 \%)$ & \\
\hline Postoperative interventional therapy & & & 0.447 \\
\hline With & $114(39.86 \%)$ & $43(35.83 \%)$ & \\
\hline Without & $172(60.14 \%)$ & $77(64.17 \%)$ & \\
\hline AJCC staging system (the 8th edition) & & & 0.362 \\
\hline Stage 1 & $21(7.34 \%)$ & $10(8.33 \%)$ & \\
\hline Stage II & $202(70.63 \%)$ & 91 (75.83\%) & \\
\hline Stage III & $63(22.03 \%)$ & $19(15.83 \%)$ & \\
\hline $\mathrm{BCLC}$ staging system & & & 0.588 \\
\hline Stage 0 & $21(7.34 \%)$ & $10(8.33 \%)$ & \\
\hline Stage A & $39(13.64 \%)$ & $14(11.67 \%)$ & \\
\hline Stage B & $199(69.58 \%)$ & $89(74.17 \%)$ & \\
\hline Stage $C$ & $27(9.44 \%)$ & $7(5.83 \%)$ & \\
\hline Early-relapse & & & 0.610 \\
\hline Presence & $115(40.21 \%)$ & $45(37.50 \%)$ & \\
\hline Absence & $171(59.79 \%)$ & 75 (62.50\%) & \\
\hline
\end{tabular}

$A L B$ albumin-bilirubin, $A L T$ alanine aminotransferase, $A S T$ aspartate aminotransferase, $A L B$ albumin, $P T$ prothrombin time, $H B s A g$ hepatitis $B$ surface antigen, $H B e A g$ hepatitis $B$ e antigen, $H C V$ - $A b$ hepatitis $C$ virus-antibody, MVI microvascular invasion, AFP alpha-fetoprotein, AJCC American Joint Committee on Cancer, BCLC

Barcelona Clinic Liver Cancer 


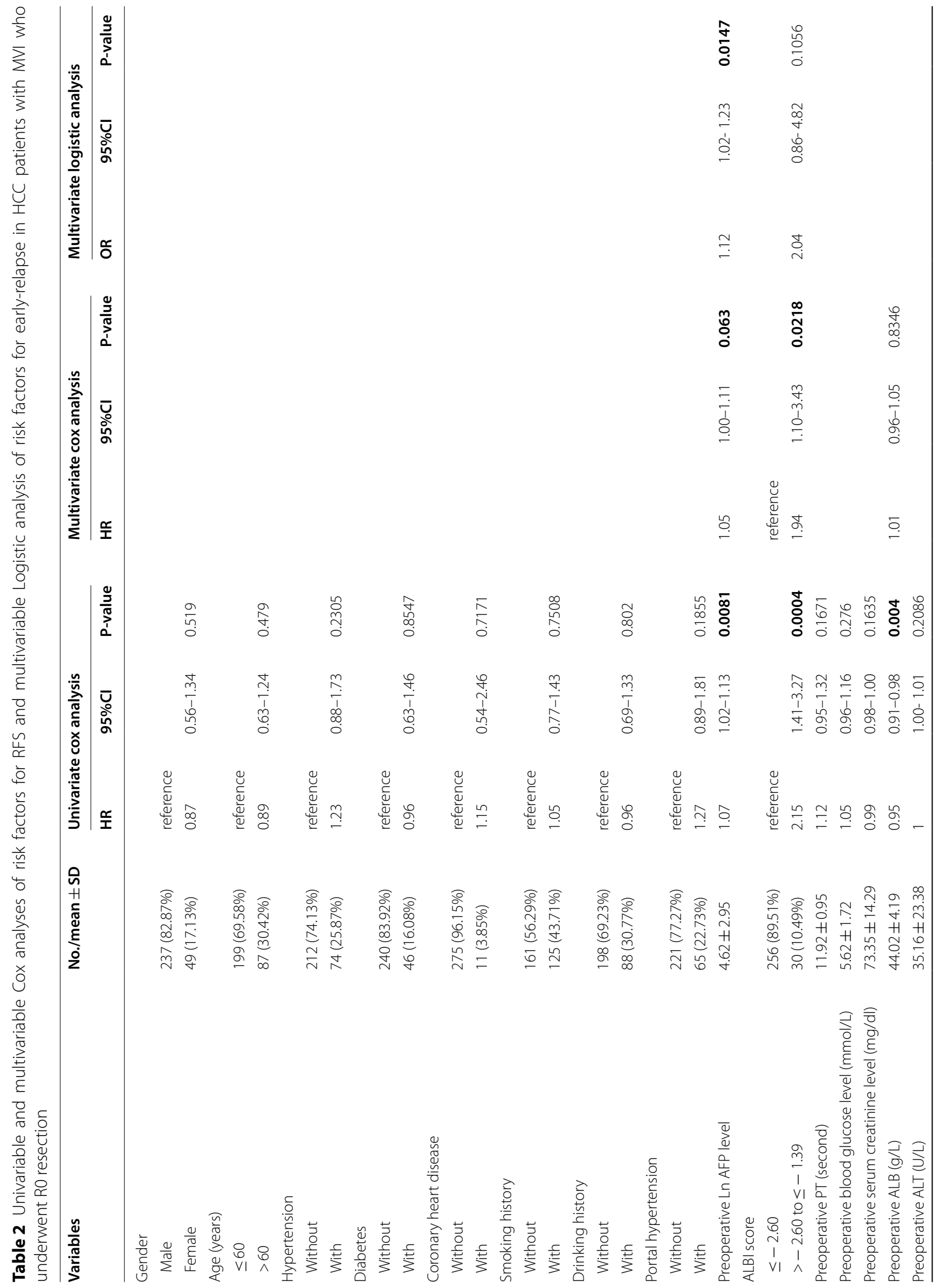




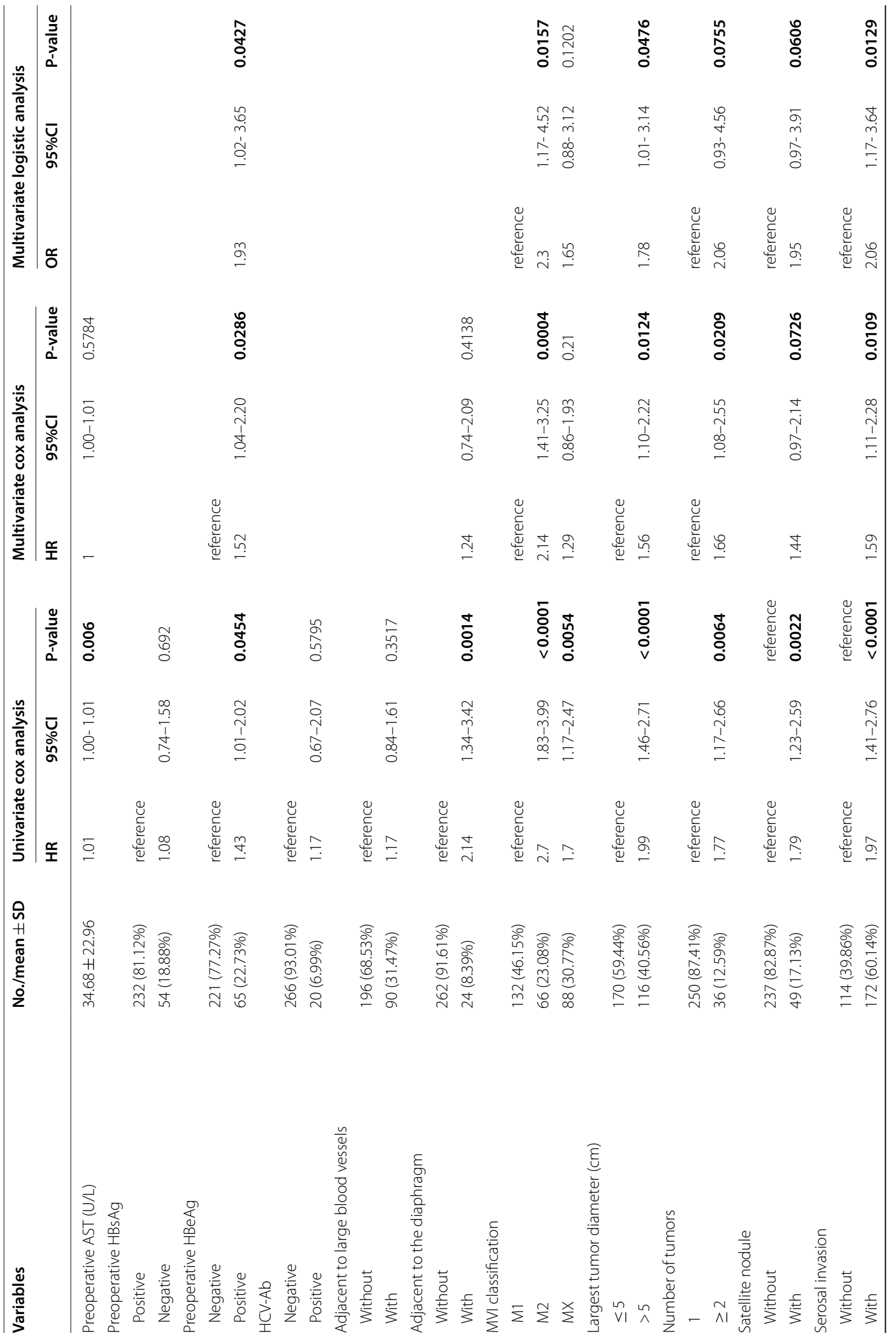




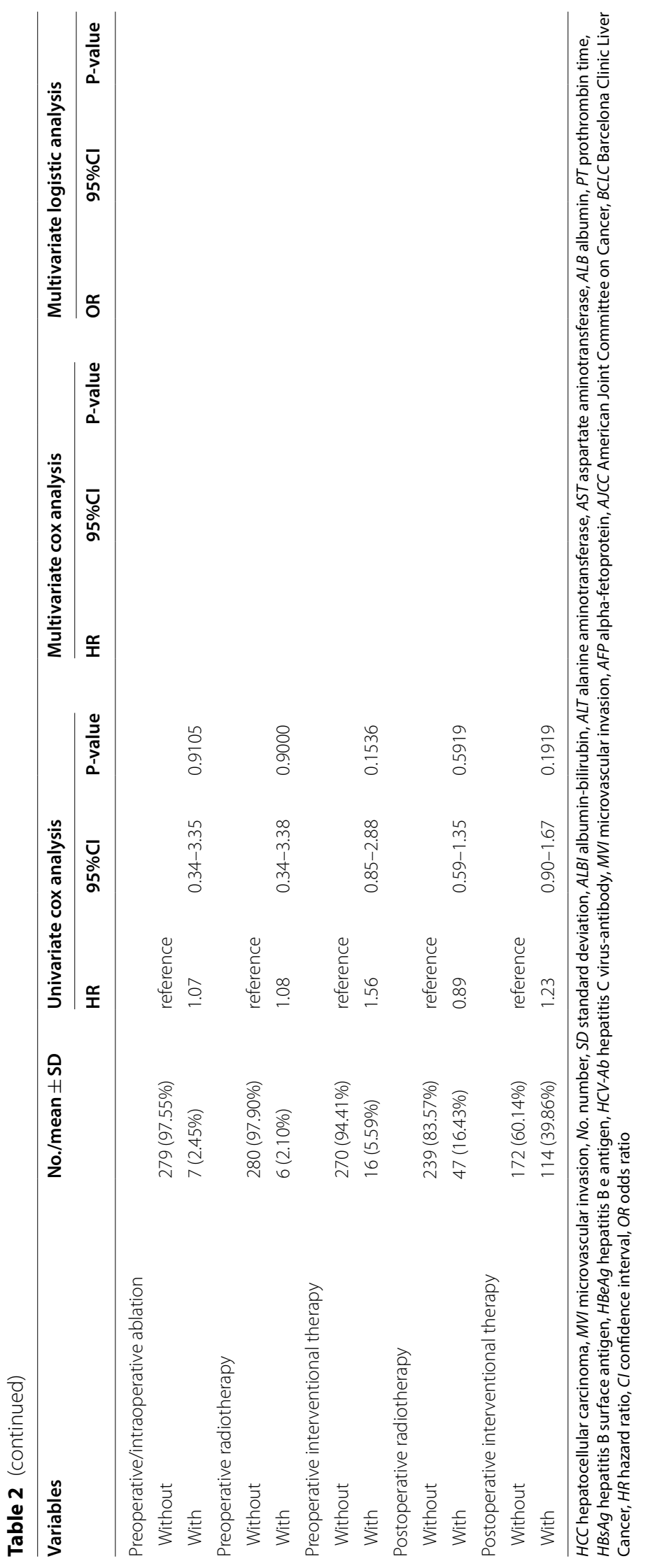


regression equation and the results were displayed in nomogram (Fig. 1). We also provided a web calculator at the website (https://zhangkaimedicalapp.shinyapps.io/ DynNomapp/) for clinicians to use this model to predict the probability of postoperative early-relapse in $\mathrm{HCC}$ patients with MVI (Additional file 2: Fig. S2). In the derivation cohort, the C-index of discrimination was 0.737 , 0.60 and 0.57 for the predictive model, AJCC and BCLC staging system, respectively. In the validation cohort, the C-index of discrimination was $0.736,0.63$ and 0.60 for the predictive model, AJCC and BCLC staging system, respectively. Collectively, the predictive model had an acceptable discriminability both in the derivation and validation cohort. The resampling was done for 1000 times using Bootstrap method to assess the model calibration. The predictive probability was consistent with

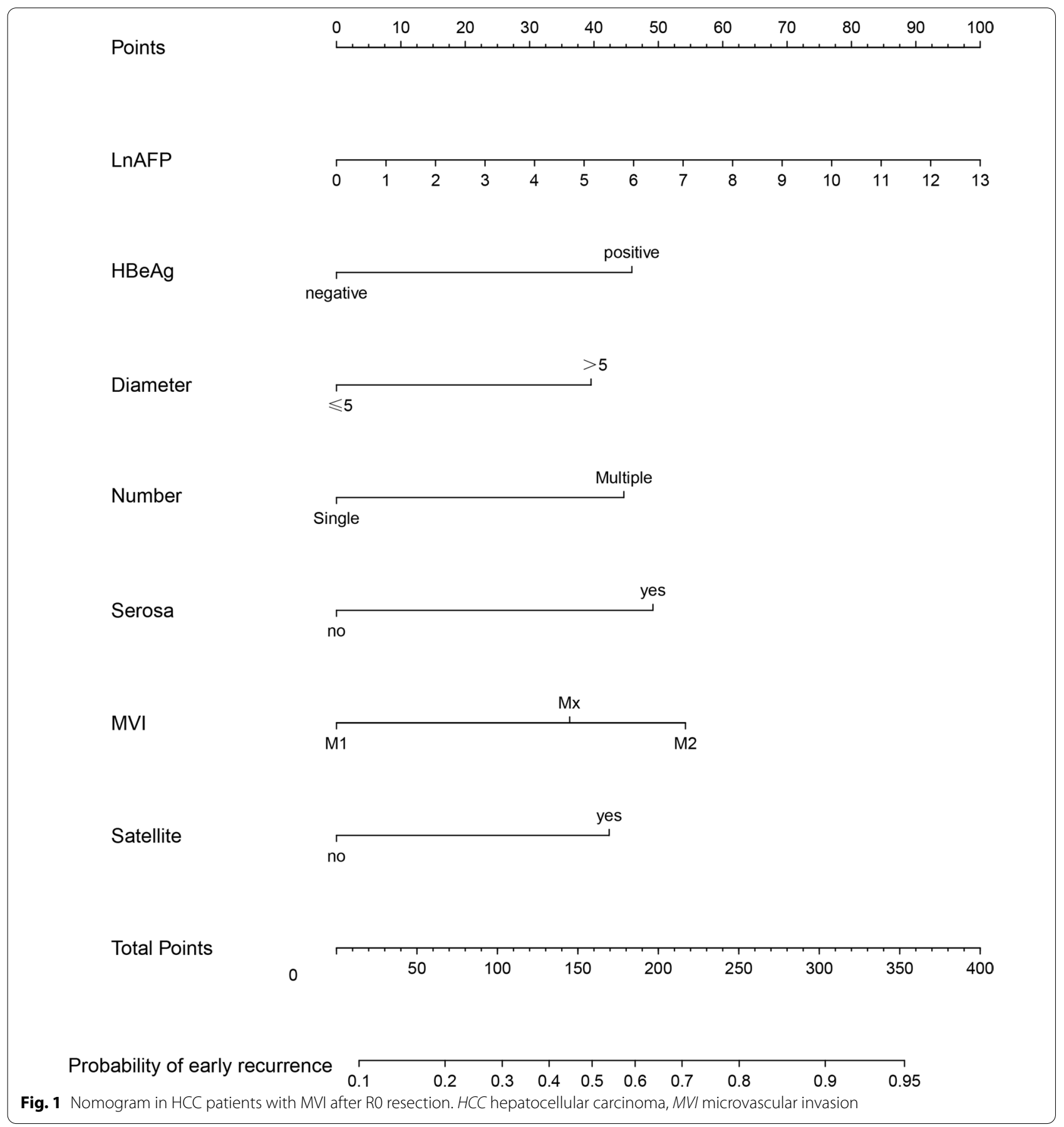


the actual probability either in the derivation or validation cohort (Additional file 3: Fig. S3). Taken together, the predictive model was feasible to accurately predict postoperative early-relapse in HCC patients with MVI.

\section{Comparison of the predictive value of the model and traditional staging systems in diagnosis of postoperative early-relapse}

DCA curve was plotted and the predictive value of the constructed model in clinical practicability was compared with that of the 8th edition AJCC staging system and BCLC staging systems. As shown by Fig. 2, in either the derivation or validation cohort, DCA curve for the predictive model was above those for traditional staging systems, which suggested that the predictive model was superior to traditional staging systems in terms of threshold probability. In order to further explore the practicable value of the predictive model, ROC curve was plotted for the predictive model, AJCC staging system and BCLC staging system and optimal cutoff value was calculated, respectively. According to the optimal cutoff value of 120 points calculated based on the Youden index, 406 patients were allocated into high- (>120 points) and low riskgroup ( $<120$ points) and actual status of postoperative early-relapse were compared between the two groups. In the derivation group, the sensitivity and specificity for the predictive model were $74 \%$ and $61 \%$ (Table 3). The sensitivity and specificity were $76 \%$ and $64 \%$ for the predictive model in the validation cohort (Table 3). Although the sensitivity of BCLC staging system was superior to that of the predictive model, its specificity was only $26 \%$ and $24 \%$ in the derivation group and validation group, respectively (Table 3). The specificity of AJCC staging system was superior to that of the predictive model, however, its sensitivity was only $32 \%$ and $31 \%$ in the derivation and validation cohort, respectively (Table 3 ). Taken together, the predictive model had the good sensitivity and specificity either in the derivation and validating cohort.

\section{Discussion}

In this study, a predictive model for postoperative earlyrelapse in HCC patients with MVI was established, which were displayed using nomogram and webpage calculator for convenient clinical application (https://zhangkaime dicalapp.shinyapps.io/DynNomapp/). In this study, 406 HCC patients with MVI were selected as subjects. Univariate and multivariate analyses were performed in the derivation cohort to identify risk factors associated with

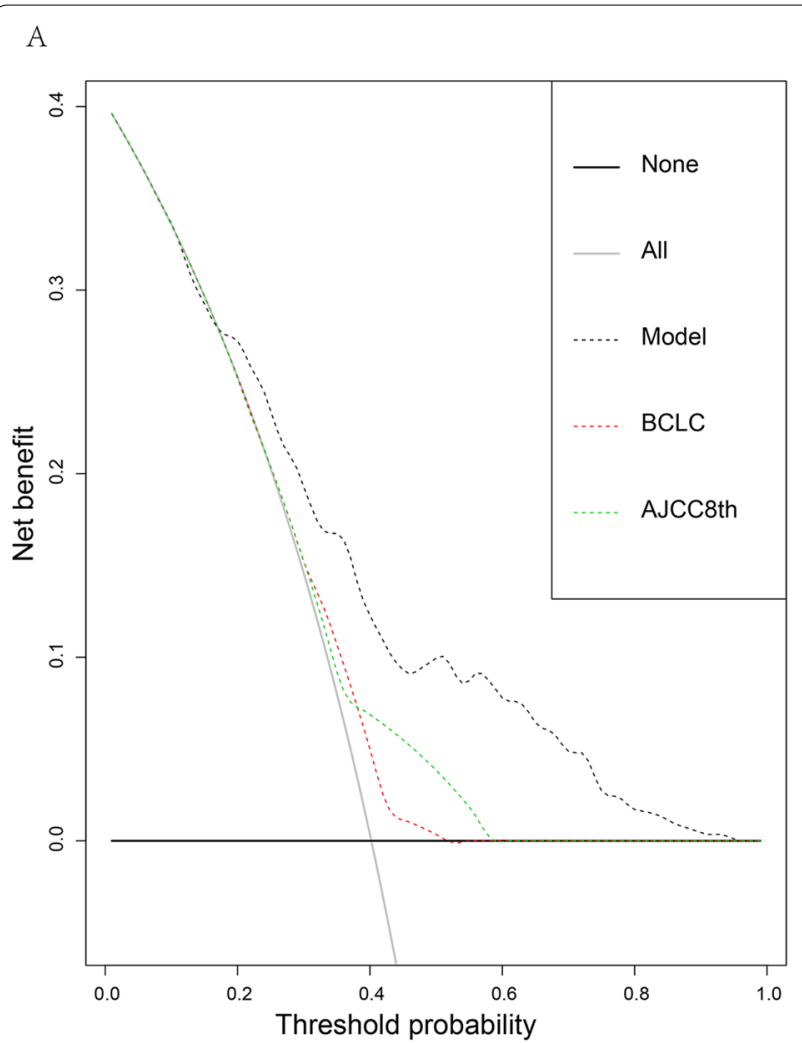

B

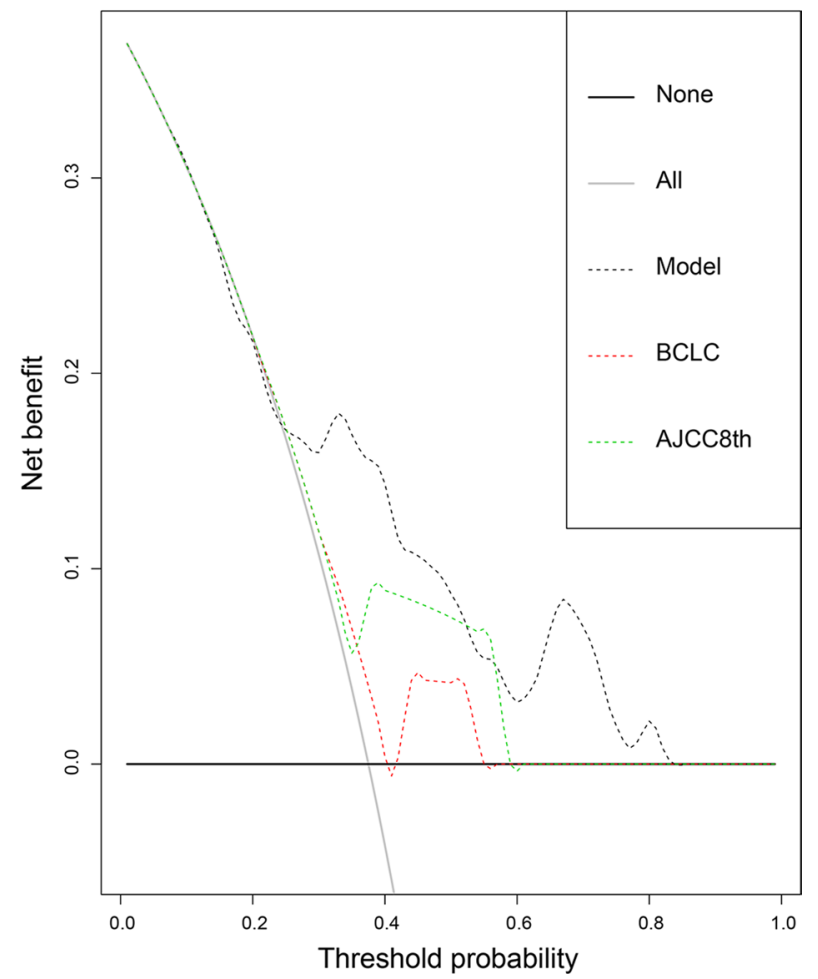

Fig. 2 Decision curve analyses in the derivation (A) and validation (B) cohort. AJCC American Joint Committee on Cancer, BCLC Barcelona Clinic Liver Cancer 
Table 3 Prognostic performance of the developed model for postoperative early-relapse in HCC patients with MVI

\begin{tabular}{|c|c|c|c|c|c|c|}
\hline \multirow[t]{2}{*}{ Performance } & \multicolumn{3}{|l|}{ Derivation cohort } & \multicolumn{3}{|l|}{ Validation cohort } \\
\hline & Predictive model & $\begin{array}{l}\text { BCLC staging } \\
\text { system }\end{array}$ & $\begin{array}{l}\text { AJCC system (the } \\
8^{\text {th }} \text { edition) }\end{array}$ & Predictive model & $\begin{array}{l}\text { BCLC staging } \\
\text { system }\end{array}$ & $\begin{array}{l}\text { AJCC system } \\
\text { (the } 8^{\text {th }} \\
\text { edition) }\end{array}$ \\
\hline Best cutoff value & 120 points & Stage A & Stage III & 120 points & Stage A & Stage III \\
\hline Sensitivity (\%) & 74 & 86 & 32 & 76 & 86 & 31 \\
\hline Specificity (\%) & 61 & 26 & 85 & 64 & 24 & 93 \\
\hline Accuracy (\%) & 66 & 61 & 64 & 68 & 47 & 70 \\
\hline
\end{tabular}

HCC hepatocellular carcinoma, MVI microvascular invasion, AJCC American Joint Committee on Cancer, BCLC Barcelona Clinic Liver Cancer

early-relapse, which were used to construct a model to predict the possibility of early-relapse in HCC patients with MVI. The discrimination, calibration and clinical usefulness of the predictive model were superior to that of traditional staging systems, such as AJCC and BCLC. According to the optimal cutoff value of 120 points, the patients were further allocated into the high- and lowrisk group, the predictive model showed the good sensitivity and specificity in distinguishing patients with high-risk early-relapse after R0 resection from those with low-risk.

The prognosis of HCC patients is mainly influenced by the following three factors: (1) Factors of patient-self, such as status of hepatitis virus infection and liver function; (2) Factors of tumor, such as diameter of tumor, MVI classification, and blood AFP level; (3) Factors of treatment, such as postoperative adjuvant treatment. In this study, 6 of all 7 risk factors associated with earlyrelapse were tumor-related factors, including preoperative blood AFP level, MVI classification, number of tumors, largest tumor diameter, the status of serosal invasion and satellite nodules. These results indicate that tumor-related factors play important roles in the postoperative early-relapse of HCC patients with MVI.

It has been documented that preoperative blood AFP level, number of tumors, and diameter of tumor are risk factors for predicting prognosis of patients [12, 14, 22, 23]. Blood AFP level might be positively related to the diameter of tumor in HCC patients, and the presence of tumor enlargement is a predictive factor for poor prognosis [24]. A previous study has reported that blood AFP level is still the independent risk factor for poor prognosis after adjusting for the presence of tumor enlargement [25]. At present, blood AFP level of $>400 \mathrm{ng} / \mathrm{mL}$ is commonly considered as the independent risk factor for poor prognosis [26]. The HCC patients with multiple tumors can be classified into patients with intrahepatic metastasis and with intrahepatic multiple primary tumors [27]. Intrahepatic metastasis indicates the disease progression on primary HCC and intrahepatic multiple primary tumors are commonly related to poor liver function. Early-relapse is more prone to occur in HCC patients with intrahepatic metastasis, and late-relapse is more prone to occur in patients with poor liver function [28]. In this study, patients were not stratified according to the status of multiple tumors. The number of tumors was identified as a risk factor with a marginally statistical difference for early-relapse might be largely due to the fact that some patients with intrahepatic multiple primary tumors were incorporated into our study.

In current viewpoints, MVI and the presence of satellite nodules are different stages during the progression of tumor. The emergence of satellite nodules indicates disease progression on HCC with MVI [20]. As shown by previous studies, MVI is an important risk factor for postoperative poor prognosis in HCC patients, and it also could predict the postoperative early-relapse [18]. The patient with high grade of MVI exhibits poor prognosis [29]. The presence of multinodular tumor is regarded as resulting from disease progression of MVI, and it is related to poor prognosis of patients [28]. It is well known that tumor size is related to the prognosis of patients. The presence of tumor enlargement predicts poor prognosis of HCC patients. The corresponding cutoff value of tumor size is used in different guidelines to predict prognosis because the correlation between tumor size and poor prognosis in patients is not in a linear manner. In this study, by referring to relevant indices recommended in the Chinese Standards for Diagnosis and Treatment of Primary Liver Cancer [17], AJCC staging system (the $8^{\text {th }}$ edition) and Hong Kong staging system for liver cancer [30], the cutoff value was set as $5 \mathrm{~cm}$ in our work. Tumor with a diameter $>5 \mathrm{~cm}$ was also identified as an independent risk factor for early-relapse in the present work.

The previous studies have demonstrated that incomplete tumor encapsulation predicts poor prognosis in HCC patients [18, 22]. In this study, the association between tumor encapsulation and prognosis was not investigated because the status of tumor encapsulation in 
most of HCC patients was unknown. The status of serosal invasion was included in the study. Serosal invasion is defined as the microscopic invasion of tumor to fibrous membrane of liver. Our work indicated that the emergence of serosal invasion was the independent risk factor for early-relapse.

Postoperative adjuvant treatment, such as postoperative interventional therapy and radiotherapy, could improve the prognosis of HCC patients with MVI [31, 32]. In our study, the postoperative interventional therapy/radiotherapy was not significantly associated with the prognosis of patients, which might be attributed to the different baseline data between patients with and without treatment and the small sample size. A study from our hospital has demonstrated that RFS is longer in patients with a narrow incision margin $(<1 \mathrm{~cm})$ after receiving postoperative radiotherapy compared with those receiving postoperative interventional therapy by using the propensity score matching method [33]. Another study also has revealed that postoperative radiotherapy improves the prognosis of patients with a narrow incision margin $(<1 \mathrm{~cm})$ [34]. M2 MVI was defined as MVI occurring at $>1 \mathrm{~cm}$ away from primary tumor or the number of MVI more than 5 [20]. Therefore, the prognosis of patients with M2 MVI may be improved after the postoperative radiotherapy because it is theoretically possible for such patients to have relatively narrow surgical margins.

In this study, among the factors of patient-self, HBeAg status was associated with postoperative early-relapse in HCC patients with MVI. A previous study has shown that $\mathrm{HBeAg}$ positivity predicts poor survival in $\mathrm{HCC}$ patients who underwent hepatectomy, which might be attributed to persistent liver injury resulting from an active virus replication [35]. Portal vein hypertension is also a poor prognostic factor in HCC patients with MVI [36]. In this study, portal vein hypertension was not significantly correlated with the postoperative early-relapse. Portal vein hypertension is one of adverse outcomes of persistent liver injury and impacts on the use of target drugs (such as Sorafenib). Portal vein hypertension and adjuvant treatments might impact on the prognosis of patients. In this study, both ALBI score for assessing the liver function and preoperative/postoperative adjuvant treatment were included. ALBI score is developed based on the data of a large sample size study in Japanese patients with different stage of HCC. The previous study has shown that only bilirubin and albumin level as nontumor-related factors impact on survival [37]. This linear predictor was calculated using the following formula: $\left(\log _{10}\right.$ bilirubin level $\left.\times 0.66\right)+$ albumin level $\times(-0.085)$ ] (the unit of bilirubin and albumin level and was $\mu \mathrm{mol} / \mathrm{L}$ and $\mathrm{g} / \mathrm{L}$, respectively). The patients were subsequently grouped into: Grade I ( $\leq-2.60$ points), Grade II ( $>-$ 2.60 to $\leq-1.39$ points) and Grade III ( $>-1.39$ points) according to the two cutoff values. In this study, ALBI score was an independent risk factor for disease relapse in HCC patients with MVI. Similar result is also shown in a previous study [38]. However, as shown by multivariate Logistic regression analysis in this study, ALBI score was not the independent risk factor for early-relapse, which might be due to the fact that poor liver function is more prone to occur in patients with late-relapse after the surgery than those with early-relapse. Similar result is shown in the previous study indicating that poor liver function increases the risk of postoperative late-relapse [28].

This study had some limitations. This study was a single-center, retrospective, and case-controlled study, which might result in the bias of our conclusions. Although the risk factors found in this study were supported by relevant studies, their internal and external truthfulness is needed to be verified. Due to a long-time span of the study, some prognostic factors were not be collected, which might result in the bias of the final conclusions. In this study, an external validation was performed in a validation group and an internal validation was performed by resampling for 1000 times using the bootstrap method in the derivation group. However, the clinical usefulness of predictive model was not validated in a real-world study. A pragmatic randomized controlled trial is needed to determine the power of the predictive model and improve the predictive model.

\section{Conclusion}

Our work indicated that MVI classification, HBeAg status, preoperative blood AFP level, number of tumors, largest tumor diameter, the status of satellite nodules and serosal invasion were independent risk factors for earlyrelapse in HCC patients with MVI after R0 resection. The predictive model established by using the abovementioned risk factors was a feasible tool to predict the possibility of early-relapse in HCC patients with MVI after R0 resection.

\section{Abbreviations}

HCC: Hepatocellular carcinoma; MVI: Microvascular invasion; AFP: Alphafetoprotein; HBeAg: Hepatitis B e antigen; HBV: Hepatitis B virus; HCV: Hepatitis C virus; SBRT: Stereotactic body radiation therapy; RFS: Relapse-free survival; OS: Overall survival; ECOG: Eastern Cooperative Oncology Group; C-index: Concordance index; AJCC: American Joint Committee on Cancer; BCLC: Barcelona Clinic Liver Cancer; ROC: Receiver operating characteristic curves; VIF: Variance inflation factor; ALBI: Albumin-bilirubin; ALB: Albumin; ALT: Alanine aminotransferase; DCA: Decision curve analyses. 


\section{Supplementary Information}

The online version contains supplementary material available at https://doi. org/10.1186/s12967-021-02940-0.

Additional file 1: Fig. S1. Flow chart of enrolled patients. HCC, hepatocellular carcinoma; MVI, microvascular invasion.

Additional file 2: Fig. S2. We provided a Web calculator (https://zhang kaimedicalapp.shinyapps.io/DynNomapp/) for predicting the probability of postoperative early-relapse in HCC patients with MVI. HCC, hepatocellular carcinoma; MVI, microvascular invasion.

Additional file 3: Fig. S3. Calibration curves in the derivation (A) and validation (B) cohort. AJCC, American Joint Committee on Cancer; BCLC, Barcelona Clinic Liver Cancer.

\section{Acknowledgements}

We would like to express our gratitude to Haiwei Du and Xiaona Han from Burning Rock Biotech for their valuable assistance in data analysis and interpretation

\section{Authors' contributions}

Study conception: JW and WR. Data collection: KZ, CT. Patient management: KZ, CT, TS. Data analyses: KZ and CT. Manuscript writing: KZ, CT, JW, WR. Manuscript revision: CT, JW, WR. All authors read and approved the final manuscript.

\section{Funding}

This work was supported by National Key Research and Development Program of China (No. 2016YFD0400604) and CAMS Innovation Fund for Medical Science (CIFMS) (CAMS-2016-I2M-3-025).

\section{Availability of data and materials}

The datasets used and/or analyzed during the current study are available from the corresponding author on reasonable request.

\section{Declarations}

\section{Ethics approval and consent to participate}

This study had been approved by Ethics Committee of Cancer Hospital of Chinese Academy of Medical Science (17-035/1290). All recruited subjects have signed the informed consent form. This study complied with the Helsinki's Declaration.

\section{Consent for publication}

Not applicable.

\section{Competing interests}

The authors declare that they have no competing interests.

Received: 29 December 2020 Accepted: 13 June 2021

Published online: 06 July 2021

\section{References}

1. Bray F, Ferlay J, Soerjomataram I, Siegel RL, Torre LA, Jemal A. Global cancer statistics 2018: GLOBOCAN estimates of incidence and mortality worldwide for 36 cancers in 185 countries. CA Cancer J Clin. 2018;68:394-424.

2. Massarweh NN, El-Serag HB. Epidemiology of hepatocellular carcinoma and intrahepatic cholangiocarcinoma. Cancer Control. 2017:24:1073274817729245

3. Li J, Zhou J, Yang PH, Xia Y, Shi YH, Wu D, et al. Nomograms for survival prediction in patients undergoing liver resection for hepatitis B virus related early stage hepatocellular carcinoma. Eur J Cancer. 2016;62:86-95.

4. Li Z, Lei Z, Xia Y, Li J, Wang K, Zhang H, et al. Association of preoperative antiviral treatment with incidences of microvascular invasion and early tumor recurrence in hepatitis B virus-related hepatocellular carcinoma. JAMA Surg. 2018;153:e182721.
5. Yoon SM, Lim YS, Park MJ, Kim SY, Cho B, Shim JH, et al. Stereotactic body radiation therapy as an alternative treatment for small hepatocellular carcinoma. PLoS One. 2013;8:e79854.

6. Schwarz RE, Abou-Alfa GK, Geschwind JF, Krishnan S, Salem R, Venook AP. Nonoperative therapies for combined modality treatment of hepatocellular cancer: expert consensus statement. HPB (Oxford). 2010;12:313-20.

7. EASL Clinical Practice Guidelines. Management of hepatocellular carcinoma. J Hepatol. 2018;69:182-236.

8. Hara K, Takeda A, Tsurugai Y, Saigusa Y, Sanuki N, Eriguchi T, et al. Radiotherapy for hepatocellular carcinoma results in comparable survival to radiofrequency ablation: a propensity score analysis. Hepatology. 2019;69:2533-45.

9. Liu PH, Hsu CY, Hsia CY, Lee YH, Huang YH, Chiou YY, Lin HC, Huo TI. Surgical resection versus radiofrequency ablation for single hepatocellular carcinoma $\leq 2 \mathrm{~cm}$ in a propensity score model: erratum. Ann Surg. 2016;263:e77.

10. Lee S, Kang TW, Song KD, Lee MW, Rhim H, Lim HK, et al. Effect of microvascular invasion risk on early recurrence of hepatocellular carcinoma after surgery and radiofrequency ablation. Ann Surg. 2019;7:89.

11. Tabrizian P, Jibara G, Shrager B, Schwartz M, Roayaie S. Recurrence of hepatocellular cancer after resection: patterns, treatments, and prognosis. Ann Surg. 2015;261:947-55.

12. Shim JH, Jun MJ, Han S, Lee YJ, Lee SG, Kim KM, et al. Prognostic nomograms for prediction of recurrence and survival after curative liver resection for hepatocellular carcinoma. Ann Surg. 2015;261:939-46.

13. Feng LH, Dong H, Lau WY, Yu H, Zhu YY, Zhao Y, et al. Novel microvascular invasion-based prognostic nomograms to predict survival outcomes in patients after R0 resection for hepatocellular carcinoma. J Cancer Res Clin Oncol. 2017:143:293-303.

14. Chan AWH, Zhong J, Berhane S, Toyoda H, Cucchetti A, Shi K, et al. Development of pre and post-operative models to predict early recurrence of hepatocellular carcinoma after surgical resection. J Hepatol. 2018:69:1284-93.

15. Lei Z, Li J, Wu D, Xia Y, Wang Q, Si A, et al. Nomogram for preoperative estimation of microvascular invasion risk in hepatitis $B$ virus-related hepatocellular carcinoma within the Milan criteria. JAMA Surg. 2016;151:356-63.

16. Lin S, Ye F, Rong W, Song Y, Wu F, Liu Y, et al. Nomogram to assist in surgical plan for hepatocellular carcinoma: a prediction model for microvascular invasion. J Gastrointest Surg. 2019;23:2372-82.

17. Wu MC, Tang ZY, Liu YY, Chen XP, Wang XH, Sun Y, et al. Standards for diagnosis and treatment of primary liver cancer. Chin J Pract Surg. 2020;40:121-38.

18. Xing $H$, Zhang WG, Cescon M, Liang L, Li C, Wang MD, et al. Defining and predicting early recurrence after liver resection of hepatocellular carcinoma: a multi-institutional study. HPB (Oxford). 2020;22:677-89.

19. Yoh T, Seo S, Taura K, Iguchi K, Ogiso S, Fukumitsu K, et al. Surgery for recurrent hepatocellular carcinoma: achieving long-term survival. Ann Surg. 2019;8:55

20. Wu MC, Tang ZY, Liu TH. Guideline for standardized pathological diagnosis of primary liver cancer. J Clin Hepatol. 2015;31:833-9.

21. Xing H, Sun LY, Yan WT, Quan B, Liang L, Li C, et al. Repeat hepatectomy for patients with early and late recurrence of hepatocellular carcinoma: a multicenter propensity score matching analysis. Surgery. 2019;74:24.

22. Zhang XP, Wang K, Wei XB, Li LQ, Sun HC, Wen TF, et al. An eastern hepatobiliary surgery hospital microvascular invasion scoring system in predicting prognosis of patients with hepatocellular carcinoma and microvascular invasion after R0 liver resection: a large-scale, Multicenter Study. Oncologist. 2019;24:e1476-88.

23. Hidaka M, Eguchi S, Okuda K, Beppu T, Shirabe K, Kondo K, et al. Impact of anatomical resection for hepatocellular carcinoma with microportal invasion (vp1): a multi-institutional study by the Kyushu Study Group of Liver Surgery. Ann Surg. 2020;271:339-46.

24. Tangkijvanich P, Anukulkarnkusol N, Suwangool P, Lertmaharit S, Hanvivatvong $\mathrm{O}$, Kullavanijaya $\mathrm{P}$, et al. Clinical characteristics and prognosis of hepatocellular carcinoma: analysis based on serum alpha-fetoprotein levels. J Clin Gastroenterol. 2000;31:302-8.

25. Matsumoto Y, Suzuki T, Asada I, Ozawa K, Tobe T, Honjo I. Clinical classification of hepatoma in Japan according to serial changes in serum alpha-fetoprotein levels. Cancer. 1982;49:354-60. 
26. Galle PR, Foerster F, Kudo M, Chan SL, Llovet JM, Qin S, et al. Biology and significance of alpha-fetoprotein in hepatocellular carcinoma. Liver Int. 2019:39:2214-29.

27. Xie DY, Fan HK, Ren ZG, Fan J, Gao Q. Identifying clonal origin of multifocal hepatocellular carcinoma and its clinical implications. Clin Transl Gastroenterol. 2019;10:e00006.

28. Yamamoto Y, Ikoma H, Morimura R, Konishi H, Murayama Y, Komatsu S, et al. Optimal duration of the early and late recurrence of hepatocellular carcinoma after hepatectomy. World I Gastroenterol. 2015:21:1207-15.

29. Roayaie S, Blume IN, Thung SN, Guido M, Fiel MI, Hiotis S, et al. A system of classifying microvascular invasion to predict outcome after resection in patients with hepatocellular carcinoma. Gastroenterology. 2009:137:850-5.

30. Tellapuri S, Sutphin PD, Beg MS, Singal AG, Kalva SP. Staging systems of hepatocellular carcinoma: a review. Indian J Gastroenterol. 2018:37:481-91.

31. Chen ZH, Zhang XP, Zhou TF, Wang K, Wang H, Chai ZT, et al. Adjuvant transarterial chemoembolization improves survival outcomes in hepatocellular carcinoma with microvascular invasion: a systematic review and meta-analysis. Eur J Surg Oncol. 2019;45:2188-96.

32. Wang L, Wang W, Rong W, Li Z, Wu F, Liu Y, et al. Postoperative adjuvant treatment strategy for hepatocellular carcinoma with microvascular invasion: a non-randomized interventional clinical study. BMC Cancer. 2020;20:614.

33. Wang L, Chen B, Li Z, Yao X, Liu M, Rong W, et al. Optimal postoperative adjuvant treatment strategy for HBV-related hepatocellular carcinoma with microvascular invasion: a propensity score analysis. Onco Targets Ther. 2019;12:1237-47.

34. Wang WH, Wang Z, Wu JX, Zhang T, Rong WQ, Wang LM, et al. Survival benefit with IMRT following narrow-margin hepatectomy in patients with hepatocellular carcinoma close to major vessels. Liver Int. 2015;35:2603-10

35. Sun HC, Zhang W, Qin LX, Zhang BH, Ye QH, Wang L, et al. Positive serum hepatitis $B$ antigen is associated with higher risk of early recurrence and poorer survival in patients after curative resection of hepatitis B-related hepatocellular carcinoma. J Hepatol. 2007;47:684-90.

36. Zhu Y, Xu D, Zhang Z, Dong J, Zhou Y, Zhang WW, et al. A new laboratorybased algorithm to predict microvascular invasion and survival in patients with hepatocellular carcinoma. Int J Surg. 2018;57:45-53.

37. Johnson PJ, Berhane S, Kagebayashi C, Satomura S, Teng M, Reeves $\mathrm{HL}$, et al. Assessment of liver function in patients with hepatocellular carcinoma: a new evidence-based approach-the ALBI grade. J Clin Oncol. 2015;33:550-8.

38. Zhang CH, Ni XC, Chen BY, Qiu SJ, Zhu YM, Luo M. Combined preoperative albumin-bilirubin (ALBI) and serum $\gamma$-glutamyl transpeptidase (GGT) predicts the outcome of hepatocellular carcinoma patients following hepatic resection. J Cancer. 2019;10:4836-45.

\section{Publisher's Note}

Springer Nature remains neutral with regard to jurisdictional claims in published maps and institutional affiliations.
Ready to submit your research? Choose BMC and benefit from:

- fast, convenient online submission

- thorough peer review by experienced researchers in your field

- rapid publication on acceptance

- support for research data, including large and complex data types

- gold Open Access which fosters wider collaboration and increased citations

- maximum visibility for your research: over 100M website views per year

At BMC, research is always in progress.

Learn more biomedcentral.com/submissions 